\section{PSICOLOGIA IBEROAMERICANA}

\section{Psicología lberoamericana}

ISSN: 1405-0943

psicología.iberoamericana@uia.mx

Universidad Iberoamericana, Ciudad de

México

México

González Jaimes, Elvira Ivone; Sanromán Aranda, Roberto; Hernández Prieto, Ma. de Lourdes Procedimientos para Disminuir los Bajos Promedios e Irregularidad Estudiantil en Universitarios Psicología Iberoamericana, vol. 19, núm. 1, enero-junio, 2011, pp. 27-38

Universidad Iberoamericana, Ciudad de México

Distrito Federal, México

Disponible en: http://www.redalyc.org/articulo.oa?id=133920896004

Cómo citar el artículo

Número completo

- Más información del artículo

Página de la revista en redalyc.org

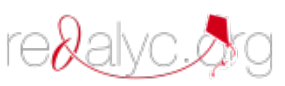

Sistema de Información Científica

Red de Revistas Científicas de América Latina, el Caribe, España y Portugal Proyecto académico sin fines de lucro, desarrollado bajo la iniciativa de acceso abierto 


\title{
Procedimientos para Disminuir los Bajos Promedios e Irregularidad Estudiantil en Universitarios
}

\author{
Procedures to Diminish the Student Irregularity as Incipient Element \\ of the Low Terminal Efficiency
}

\author{
Elvira Ivone González Jaimes \\ Roberto Sanromán Aranda \\ Ma. de Lourdes Hernández Prieto* \\ Centro Universitario Valle de México, Estado de México, México
}

\section{RESUMEN}

Se trata de un estudio comparativo de dos procedimientos, aplicados para disminuir la cantidad de estudiantes universitarios con bajos promedios e inscripción irregular. El diseño fue cuasiexperimental para dos grupos experimentales: tratamiento Psicoterapia Breve Espejo 1(PBE) y Estrategia de Aprendizaje de Mapas Conceptuales 2(EAMC); con cuatro mediciones (pre test, post test, seguimiento de seis y 12 meses). La población estuvo compuesta por 2510 participantes y fue una muestra condicionada (bajo rendimiento e inscripción irregular) de 483 estudiantes pertenecientes a 10 carreras universitarias, de ambos géneros, y de entre 18 a 32 años de edad. Los materiales fueron los siguientes: Inventario multifásico de la personalidad de Minnesota-2 y Cuestionario de estrategias de aprendizaje en universitarios. Resultados: El factor Impacto por procedimiento, 1(PBE) disminuyo un 20.5\% y 2(EAMC) disminuyo un 13\%. El factor Impacto por tiempo, 1(PBE) tiene mayor efecto a largo plazo. El factor Impacto por carrera, $1(\mathrm{PBE})$, se presenta $91 \%$ en la carrera de Ingeniería Industrial con aplicabilidad concreta, y 2(AEMC) se presenta 52\% en la Licenciatura en Administración, con aplicabilidad abstracta.

Descriptores: Bajo rendimiento, irregularidad estudiantil, psicoterapia breve espejo, estrategia de aprendizaje de mapas conceptuales.

\section{ABSTRACT}

Study applies and compares of two procedures for diminish student's low averages and student university irregularity. Quasi experimental design and comparative applied for two experimental groups: Brief Psychotherapy Mirror Treatment 1 (BPM) and Learning strategy of Conceptual Maps 2 (LECM); four measurements (pre test, post test, follow-up six and 12 months.). Population of 2510 from ten careers, generate both; 18 to 32 years of age. The sample of 483 students with under average and with condition irregular inscription. Materials. - Use the Minnesota multiphasic of personality inventory-2 and Learning strategies questionnaire for university students. Results: The factor impact for procedure, 1 (PBE) I diminish $20.5 \%$ and 2 (EAMC) diminish $13 \%$. The factor impact per time, 1 (PBE) has major long-term effect. The factor impact for career, 1 (PBE), one presents $91 \%$ in the career of Industrial Engineering with concrete applicability, and 2 (AEMC) one presents $52 \%$ in the career in Administration, with abstract applicability.

Key words: Low averages, university irregularity, brief psychotherapy mirror, learning strategy of conceptual maps

\footnotetext{
* Para correspondencia: Centro Universitario Valle de México, Universidad Autónoma del Estado de México, Dirección: Bulevar Universitario S/N, Predio San Javier, Municipio de Atizapán de Zaragoza, Estado de México, C.P. 54500. Elvira Ivone González Jaimes, e-mail: ivonegj@hotmail.com, Teléfonos: (55) 537917 45, (044) 55134069 50, Fax 58270703. Roberto Sanromán Aranda; email: r_sanroman31@hotmail.com y Ma. de Lourdes Hernández Prieto, email: ulahp2003@yahoo.com.mx.
} 


\section{INTRODUCCIÓN}

Entre los problemas más complejos y frecuentes que enfrentan las instituciones de estudios superiores oficiales, que ocupan un $58.34 \%$ de la población universitaria en nuestro país, son: el bajo rendimiento y la irregularidad estudiantil como base en el rezago estudiantil, lo que incide en la deserción escolar, y afecta tanto el nivel de calidad educativa, como la eficiencia terminal (Programas Institucionales de Tutoría, 2002). En una investigación que proviene de la Universidad Veracruzana se indica que 25 de cada 100 estudiantes que ingresan al nivel universitario abandonan sus estudios. En una proporción de un 36\% los primeros dos semestres, hasta alcanzar el $46 \%$ al término del periodo de formación (Romo, 2005).

Ante la problemática que viven las universidades oficiales en México, la Universidad Autónoma del Estado de México, plantel Centro Universitario Valle de México (CU UAEM, Valle de México), dentro del Programa de Tutoría Institucional Académica (ProInsta) ha creado el Módulo de Desarrollo Humano Integral (MDHI), que contiene proyectos innovadores que van acorde con los retos que enfrenta el mantenerse a la vanguardia del conocimiento científico, tecnológico y humanista.

El Módulo de Desarrollo Humano Integral, en el periodo (2009-A), se dio a la tarea de hacer un curso de 40 horas con Tutores Académicos del C.U UAEM Valle de México para que éstos dieran a conocer sus vivencias cotidianas sobre los factores que influían sobre los bajos promedios, ${ }^{1}$ aunados a la irregularidad estudiantil, ${ }^{2}$ considerando a estos dos factores como un bloque que funge como una variable dependiente multifactorial. Se encontraron principalmente dos grupos: un grupo de factores emocionales y otro de factores de aprendizaje.

En el grupo de factores emocionales cotidianos se encontró: baja autoestima, capacidad de logro, ansiedad y depresión.

\footnotetext{
${ }^{1}$ Programas Institucionales de Tutoría. (2002), señala: Presentan el bajo rendimiento escolar todos los estudiantes de tengan un promedio final semestral registrado entre rango de calificaciones 6 a 7.5 puntos.

${ }^{2}$ Reglamento de Facultades y Escuelas Profesionales (2009), Arts. 107111, señala: Alumno irregular es: todo alumno que tengan registrado en actas ante Departamento de Control Escolar calificación menor a 6 puntos, $\mathrm{n} / \mathrm{p}=$ No presentó, $\mathrm{s} / \mathrm{d}=$ Sin derecho, por no contar con el mínimo de asistencias consideradas en el o cuando no presentó alguna evaluación parcial.
}

En el de factores de aprendizaje cotidianos se encontró: falta de técnicas adecuadas para la adquisición, codificación, integración y recuperación de conocimientos.

Para confirmar dicha percepción realizada por los Tutores Académicos se realizó una evaluación psicométrica a 2,842, estudiantes del C.U UAEM Valle de México en el periodo 2009-A con el Inventario Multifásico de la Personalidad de Minnesota-2 (MMPI-2) escalas de contenido, en el que se obtuvo: estudiantes con nivel alto $(\mathrm{T}<65)$ : Ansiedad $(\mathrm{ANS})=1045 ; 36.37 \%$, Baja autoestima $(\mathrm{BAE})=532 ; 18.51 \%$ y Depresión $(D E P)=305 ; 10.61 \%$ (Lucio, 1995). También se aplicó el Cuestionario de Estrategias de Aprendizaje en Universitarios CEA-U, con el que se obtuvo: estudiantes con bajo niveles de calificación en las escalas: Estrategias Motivacionales $(\mathrm{EM})=564 ; 19.63 \%$ y Estrategias Cognitivas $(\mathrm{EC})=732 ; 25.47 \%$ (Martín, García, Torbay \& Rodríguez, 2007).

En el Reglamento de Facultades y Escuelas Profesionales (2009), en sus Arts. 107-111, se da la pauta para tener registro como estudiantes regulares e irregulares. Observándose en el C.U. Valle de México, periodo académico 2010 A, 2510 estudiantes (población inscrita) se contempla un rezago escolar de $7 \%$ y una deserción de $14 \%$; ambos índices son con frecuencia determinados por el $19 \%$ de estudiantes con bajo promedio e inscripción irregular, lo que afecta directamente a la eficiencia terminal. Estos estudiantes se encuentran en riesgo de ser eliminados del sistema educativo, pues dicha normatividad orilla a los estudiantes a que se regularicen o se salgan de dicho sistema ${ }^{3}$.

Lo que coadyuva a incrementar el índice de deserción escolar y en el mejor de los casos, el del rezago escolar, y afecta de manera significativa en la eficiencia terminal, que en parte, son los factores que originan la búsqueda, cambio, renovación de planes, programas, tratamientos y estrategias para promover mejoras en la educación. (Programas Institucionales de Tutoría, 2002). Lo que lleva a coincidir con la idea de que "se atribuye a la educación, la misión de permitir a todos los ciudadanos el hacer fructificar todos sus talentos y todas sus capacidades de creación..." (Delors, 1997). Por lo tanto, este estudio realiza un procedimiento psi-

${ }^{3}$ Información obtenida del Sistema de Gestión Escolar perteneciente al Departamento de Control Escolar (2010). C.U. UAEM, Valle de México. 
cológico como estrategia de aprendizaje apropiado, basándose en la psicometría antes mencionada (periodo 2009-A), para disminuir el bajo rendimiento escolar e irregularidad estudiantil.

\section{1) Procedimiento psicológico}

Según lo encontrado por el MMPI-2 en las escalas de contenido Ansiedad (ANS), Baja Autoestima (BAE) y Depresión (DEP), estos instrumentos refieren principalmente una hipersensibilidad ante la presión ambiental, actitudes y pensamientos negativos hacia sí mismo, el miedo a no alcanzar sus creencias, "lo que deber ser," así como sentimientos de inutilidad y desesperanza (Lucio, 1995).

Con base en lo anterior se encontró que existen varios estudios que nos informan sobre la relación entre la estabilidad emocional y el aprendizaje, como lo mencionan Adlaf, Gliksman, Demers y Newton-Taylor (2001) del Departamento de Salud Pública de Toronto, Canadá, en el que hallaron que, en una muestra de 7,800 estudiantes de 16 universidades, el 30\% presentaba estrés psicológico elevado, lo cual afectaba su rendimiento académico. En seis publicaciones, González-Forteza, et al., (2008) observaron, en estudiantes mexicanos, que el $47 \%$ de quienes presentaban depresión mayor tenían bajo aprovechamiento y alto abandono escolar.

Arco, et al., (2005) realizaron un estudio sobre terapia breve con tres estudiantes universitarios que tenían problemas de rendimiento académico y ansiedad, en el que se observó la mejora en su vida general y en el rendimiento escolar en la evaluación de seguimiento de nueve meses.

El procedimiento psicológico que se aplica en este estudio es el de Tratamientos de Psicoterapia Breve Espejo, el cual disminuye la ansiedad, depresión y baja autoestima, los cuales afectan en gran medida a los estudiantes y los llevan a adoptar comportamientos poco adaptativos que repercuten en su desempeño en general (González \& Turnbull, 2003; 2007).

El Tratamiento Psicoterapia Breve Espejo (PBE), pretende realizar cambios cognitivos en la adquisición de conocimientos a través de: 1) enseñar habilidades para bajar los síntomas ansiosos depresivos ante el manejo de situaciones novedosas y estresantes; 2) fomentar el autocontrol de síntomas ansioso depresivos para mejorar la adquisición y recuperación de cono- cimientos; 3) mejorar la autoestima y la capacidad de decisión para la ejecución de actividades (González \& Turnbull, 2003; 2007; González \& López, 2008).

Para alcanzar sus objetivos, el tratamiento PBE utiliza tres tipos de técnicas:

a) Técnica psico-corporal. Promueve el equilibrio de la energía psico-corporal y la conciencia de los síntomas ansiosos depresivos ante situaciones académicas difíciles de manejar (Campos y Gutiérrez, 2001).

b) Técnica cognitiva-conductual. Ayuda analizar los pensamientos a conductas asociadas la Adquisición, Codificación y Recuperación para: aceptación y manejo de los síntomas ansioso depresivos que afectan la motivación, autoestima y autocontrol de situaciones estresantes (Borkowski, 1995).

c) Técnica integrativa de autocontrol. Facilita al estudiante a la autoobservación y la autoevaluación, lo cual activa los impulsos de auto conservación y crecimiento integral del yo (González \& Turnbull, 2003; 2007; González \& López, 2008).

\section{2) Procedimiento de aprendizaje}

De acuerdo con las escalas del $C E A-U$, encontramos bajo índice en las escalas de Estrategias Motivacionales y en las Estrategias Cognitivas; estas escalas se refieren al deseo intrínsico de realizar las actividades, las cuales se reflejan en: a) disposición y constancia ante el aprendizaje y b) sencibilizaciòn y restructuración de la información (Martín et al., 2007). Los estudiantes del presente estudio (10 carreras en áreas de ciencias sociales y exactas) presentan necesidades específicas de aprendizaje, las cuales son: Adquisición, Codificación o Almacenamiento y Recuperación base indispensable para el aprendizaje, como lo señalan Valle, et al., (2006).

El procedimiento de aprendizaje que propone el presente estudio es la Estrategia de Aprendizaje de Mapas Conceptuales (EAMC), ya que tiene la capacidad de satisfacer las necesidades de aprendizaje a través de los pasos para el procesamiento de información requerido, como exponen los autores Cañas, et al., (2002).

En la Estrategia de Aprendizaje de Mapas Conceptuales se obtiene el aprendizaje significativo, a través 
de aprender a relacionar los nuevos conocimientos de manera organizada y sustancial, encadenándolos con aprendizajes previos. Lo que ha demostrado gran utilidad en las áreas de aprendizaje social y exacto, como lo requiere nuestro estudio, de acuerdo con la investigación realizada por Ausubel, Novak y Hanesian (1997). La Estrategia de Aprendizaje de Mapas Conceptuales se origina en la técnica de este tipo de mapa, desarrollado por Novak en 1991 como estrategia para guiar a los estudiantes a aprender y a organizar materiales de aprendizaje, o bien para encontrar los procedimientos a seguir en la resolución de problemas.

En investigaciones recientes se señala que el mapa conceptual es la principal herramienta metodológica, usada por personas de los más variados niveles y áreas de aprendizaje (Cuevas, 2003).

Los mapas conceptuales contienen tres elementos fundamentales: a) Los conceptos, que son palabras o signos con los que se expresan regularidades; b) Las proposiciones, que son dos o más términos conceptuales unidos por palabras de enlace para formar una unidad semántica; c) Las palabras de enlace, que sirven para relacionar los conceptos verbales y términos matemáticos (Febles \& Estrada, 2002).

De acuerdo con lo anterior podemos concluir que existen factores emocionales y de aprendizaje negativos que afectan a los estudiantes, que tienen como consecuencia la obtención de bajos promedios e inscripción irregular, por lo que este estudio propone dos procedimientos que disminuyan dichos factores para aumentar el rendimiento académico.

\section{MÉTODO}

\section{Objetivos}

1) Conocer las diferencias significativas entre los dos procedimientos, en cuanto a la disminución de estudiantes con bajos promedios e irregulares, para saber cuál de ellos obtuvo más impacto.

2) Conocer las diferencias significativas entre los dos procedimientos, en cuanto a la disminución de factores emocionales y de aprendizaje, para saber cuál de ellos obtuvo más impacto.

3) Conocer cuál de los dos procedimientos impactó más a qué carrera, en cuanto a disminución de estudiantes con bajos promedios e irregulares.
4) Conocer qué carrera aumentó más en su promedio escolar con ambas intervención.

Diseño. Diseño cuasi-experimental, de campo para dos grupos experimentales tratamiento Psicoterapia Breve Espejo 1 (PBE) y Estrategia de Aprendizaje de Mapas Conceptuales 2 (EAMC), longitudinal con tres mediciones (pre-test, post-test y seguimiento 6.).

\section{Muestra}

Se obtuvieron 2510 estudiantes pertenecientes a 10 carreras (ciencias exactas y humanas) del C.U. UAEM Valle de México; de ambos géneros y de entre 18 a 32 años de edad.

Se seleccionó al $19 \%$ de la población (483 estudiantes) porque ellos cumplían con la condición de tener bajo promedio, menor a 7.6, e inscripción de irregular. Sus características fueron: edad promedio 21.7 años; géneros masculino 59.8\% y femenino $39.2 \%$ y con promedio semestral de 6.83 .

\section{Instrumentos}

\section{Inventario Muntifásico de la Personalidad de Minnesota-2}

La nueva versión del MMPI-2 fue estandarizada para habla española por Lucio (1995), en población universitaria mexicana con confiabilidad $\alpha=.77$ y con altos niveles de validez interna y externa. El Test MMPI-2 contiene: 10 escalas clínicas, 12 suplementarias, no utilizadas en esta investigación, y 15 escalas de contenido, de las cuales utilizamos tres: Ansiedad (ANS), $\alpha=.78$; Baja Autoestima (BAE), $\alpha=.77$ y Depresión (DEP), $\alpha=.79$. La prueba de MMPI-2 fue utilizada para hacer la selección de la muestra. Sólo se seleccionó a estudiantes que tuvieron puntajes " $\mathrm{T}$ " > 65 en las Escalas de Contenido: ANS, BAE y DEP,

\section{Cuestionario de Estrategias de Aprendizaje para Universitarios (CEA-U)}

El Cuestionario de Estrategias de Aprendizaje para Universitarios (CEA-U) de Martín, García, Torbay y Rodríguez (2007) consta de un total de 57 ítems con escala de respuesta tipo Likert, donde 1 significa nada; 2 , poco; 3 , algunas veces; 4 , a menudo y 5 , siempre. El CEA-U consta de tres subescalas: 1) Estrategias 
Motivacionales (27 ítems); coeficiente de fiabilidad $\alpha=.82$; rangos de la escala: alto $=135-108$, medio $=107-81$, bajo >81; con cinco sub-escalas: implicación, asociación en positivo, aplicabilidad, auto-esfuerzo y aproximación gradual. 2) Estrategias Cognitivas (22 ítems); coeficiente de fiabilidad $\alpha=.73$; rangos de la escala: alto $=110-88$, medio $=87-66$, bajo $>66$; con cuatro subescalas: organización, elaboración generativa, elaboración de anclaje y memorización. 3) Estrategias de Control (ocho ítems); coeficiente de fiabilidad $\alpha=.81$; rangos de la escala: $=40-32$, medio $=31-24$, bajo $>24$; con dos sub-escalas: planificación y revisión.

\section{Procedimiento}

1. Se seleccionó sólo a los estudiantes de bajo promedio (<7.6) e irregulares de las 10 carreras del C.U. UAEM Valle de México.

2. Se aplicó Pre-test de MMPI-2, Escalas de Contenido DEP, BAE y ANS y Cuestionario de Estrategias de Aprendizaje en Universitarios CEA-U a 483 estudiantes de bajo promedio e irregulares.

Se formaron dos grupos experimentales aleatorios para ser asignados al Tratamiento Psicoterapia Breve Espejo, 1(PBE) y la Estrategia de Aprendizaje de Mapas Conceptuales 2 (EAMC). Se formaron así 20 subgrupos para cada procedimiento.

Condiciones éticas. Los 20 subgrupos recibieron iguales condiciones de procedimiento, como lo mencio- nan los principios éticos para las investigaciones médicas y terapéuticas en seres humanos por la Declaración de Helsinki de la Asociación Médica Mundial (2008).

3. Se aplicó a todos los estudiantes el formato de consentimiento y responsabilidad para ser firmada, la cual tendrá uso confidencial. Los procedimientos fueron realizados dentro de áreas y horarios escolares en forma grupal. La ejecución de los procedimientos tuvo comienzo y término similar. Los ejecutores fueron 10 licenciados en Psicología y 10 licenciados en Pedagogía; quienes fueron previamente instruidos en la aplicación de los mismos. El tiempo de aplicación de cada uno fue de 12 semanas; una sesión por semana de dos horas. El tiempo total de cada procedimiento fue de $24 \mathrm{hrs}$.

4. Se aplicó el post-test para medir el impacto que tuvieron ambos procedimientos.

5. Se realizaron los seguimientos durante seis y 12 meses de los grupos 1 (PBE) y 2 (EAMC), para observar el sostenimiento del impacto de los procedimientos.

6. Se aplicó el post-test y seguimiento por seis y 12 meses de las pruebas estadísticas Prueba t de student, y prueba $\mathrm{F}$ de Fisher, para conocer si existen varianzas significativas entre los dos, grupos $\mathrm{p}<0.050$.

\section{RESULTADOS}

1. Se realizó análisis descriptivo de la muestra para conocer las características de la misma (ver Tabla 1).

Tabla 1. Características sociodemográficas de la muestra

\begin{tabular}{|c|c|c|c|c|c|c|}
\hline Plan educativo & $\begin{array}{l}\text { Población, } \\
\text { total de } \\
\text { estudiantes } \\
\text { inscritos }\end{array}$ & $\begin{array}{c}\text { Muestra, } \\
\text { estudiantes } \\
\text { irregulares } \\
\text { con promedio } \\
<7.6\end{array}$ & $\begin{array}{l}\text { Edad promedio } \\
\text { de la muestra }\end{array}$ & $\begin{array}{l}\text { Género } \\
\text { masculino }\end{array}$ & $\begin{array}{l}\text { Género } \\
\text { Femenino }\end{array}$ & $\begin{array}{l}\text { Promedio de } \\
\text { calificaciones } \\
\text { de la muestra }\end{array}$ \\
\hline LAF & 129 & 20 & 22.05 & 13 & 7 & 6.710 \\
\hline LEC & 108 & 27 & 21.03 & 16 & 11 & 6.838 \\
\hline LRI & 280 & 35 & 20.09 & 19 & 16 & 7.182 \\
\hline LCN & 268 & 60 & 21.08 & 38 & 22 & 6.744 \\
\hline LIA & 311 & 75 & 21.07 & 43 & 32 & 6.868 \\
\hline LDE & 568 & 81 & 22.01 & 49 & 32 & 6.831 \\
\hline IIN & 139 & 22 & 22.11 & 12 & 10 & 6.819 \\
\hline
\end{tabular}




\begin{tabular}{|c|c|c|c|c|c|c|}
\hline Plan educativo & $\begin{array}{c}\text { Población, } \\
\text { total de } \\
\text { estudiantes } \\
\text { inscritos }\end{array}$ & $\begin{array}{c}\text { Muestra, } \\
\text { estudiantes } \\
\text { irregulares } \\
\text { con promedio } \\
<7.6\end{array}$ & $\begin{array}{c}\text { Edad promedio } \\
\text { de la muestra }\end{array}$ & $\begin{array}{c}\text { Género } \\
\text { masculino }\end{array}$ & $\begin{array}{c}\text { Género } \\
\text { Femenino }\end{array}$ & $\begin{array}{c}\text { Promedio de } \\
\text { calificaciones } \\
\text { de la muestra }\end{array}$ \\
\hline ISC & 296 & 79 & 22.01 & 49 & 30 & 6.808 \\
\hline ICO & 149 & 35 & 21.08 & 22 & 21 & 6.746 \\
\hline LAM & 262 & 49 & 22.04 & 28 & 194 & 6.806 \\
\hline Total & 2510 & 483 & & 21.799 & & 6.89 \\
\hline
\end{tabular}

Donde: LAF, Licenciado en Actuaría Financiera; LEC, Licenciado en Economía; LRI, Licenciado en Relaciones Internacionales; LCN, Licenciado en Contaduría; LIA, Licenciado en Informática Administrativa; LDE, Licenciado en Derecho, IIN, Ingeniería Industrial, ISC, Ingeniería en Sistemas Computacionales; ICO, Ingeniería en Computación; LAM, Licenciado en Administración.

Para saber si la asignación de los estudiantes a los grupos era semejante se utilizó un análisis intergrupal a través de la prueba de $t$ de Student (muestras independientes) donde no se observaron diferencias significativas en el Pre-test.
2. Prueba $t$ de student y prueba F de Fisher para dos muestras independientes (grupos de estudiantes). Observándose sólo en seguimiento durante 12 meses $\underline{F}(321)=2.45$, (ver Tabla 2).

Tabla 2. Diferencias significativas entre ambos procedimientos

\begin{tabular}{|c|c|c|c|c|c|c|c|}
\hline \multirow{2}{*}{$\begin{array}{c}\text { Variable } \\
\text { independientes } \\
\text { tratamientos }\end{array}$} & \multirow{2}{*}{$\begin{array}{c}\text { Variables } \\
\text { dependientes }\end{array}$} & \multirow{2}{*}{ Evaluación } & \multicolumn{3}{|c|}{ Prueba t student intragrupal } & \multicolumn{2}{|c|}{$\begin{array}{c}\text { Prueba F Fisher, } \\
\text { intergrupal }\end{array}$} \\
\hline & & & $t$ & $g l$ & $p>0.05$ & Leven F & $p<0.05$ \\
\hline $\begin{array}{c}\text { Estrategia de } \\
\text { aprendizaje } \\
\text { de Mapas } \\
\text { Conceptuales y } \\
\text { Psicoterapia Breve } \\
\text { Espejo }\end{array}$ & $\begin{array}{c}\text { Bajo rendimiento< } \\
7.6 \text { e irregularidad } \\
\text { estudiantil }\end{array}$ & $\begin{array}{c}\text { Seguimiento } 12 \\
\text { meses }\end{array}$ & 1.34 & 321 & 0.0612 & 2.45 & 0.0492 \\
\hline
\end{tabular}


3. Prueba $t$ de student y prueba $F$ de Fisher para dos muestras independientes, impacto de procedimientos medidos a través de escalas de Contenido de MMPI-2, (ver Tabla 3).
4. Prueba t de student y prueba F de Fisher para dos muestras independientes, impacto de procedimientos en variable en subescalas del CEA-U (ver Tabla 4).

Tabla 3. Diferencias significativas entre factores emocionales de ambos procedimientos

\begin{tabular}{|c|c|c|c|c|c|c|c|}
\hline \multirow{2}{*}{$\begin{array}{l}\text { Variables } \\
\text { independientes } \\
\text { procedimientos }\end{array}$} & \multirow{2}{*}{$\begin{array}{c}\text { Variable } \\
\text { dependiente } \\
\text { escalas de } \\
\text { Contenido }\end{array}$} & \multirow{2}{*}{ Evaluación } & \multicolumn{3}{|c|}{ Prueba $t$ student intragrupal } & \multicolumn{2}{|c|}{$\begin{array}{c}\text { Prueba F Fisher, } \\
\text { intergrupal }\end{array}$} \\
\hline & & & $t$ & $g l$ & $p>0.05$ & Leven $F$ & $p<0.05$ \\
\hline \multirow{2}{*}{$\begin{array}{c}\text { Psicoterapia Breve } \\
\text { Espejo }\end{array}$} & Depresión & Post test & 7.14 & 68 & 0.125 & 8.083 & 0.045 \\
\hline & Baja autoestima & $\begin{array}{c}\text { Seguimiento } 12 \\
\text { meses }\end{array}$ & 7.67 & 25 & 0.24 & 12.62 & 0.048 \\
\hline \multirow{2}{*}{$\begin{array}{l}\text { Estrategia de } \\
\text { aprendizaje } \\
\text { de Mapas } \\
\text { Conceptuales }\end{array}$} & \multirow{2}{*}{ Ansiedad } & $\begin{array}{c}\text { Seguimiento } 6 \\
\text { meses }\end{array}$ & 11.32 & 98 & 0.055 & 18.23 & 0.025 \\
\hline & & $\begin{array}{c}\text { Seguimiento } 12 \\
\text { meses }\end{array}$ & 5.72 & 45 & 0.32 & 24.48 & 0.018 \\
\hline
\end{tabular}

Tabla 4. Diferencias significativas entre factores de aprendizaje de ambos procedimientos

\begin{tabular}{|c|c|c|c|c|c|c|c|}
\hline \multirow{2}{*}{$\begin{array}{c}\text { Variables } \\
\text { independientes } \\
\text { procedimientos }\end{array}$} & \multirow{2}{*}{$\begin{array}{c}\text { Variable } \\
\text { dependiente } \\
\text { CEA-U Sub- } \\
\text { escalas }\end{array}$} & \multirow{2}{*}{ Evaluación } & \multicolumn{3}{|c|}{ Prueba $t$ student intragrupal } & \multicolumn{2}{|c|}{$\begin{array}{c}\text { Prueba F Fisher } \\
\text { intergrupal }\end{array}$} \\
\hline & & & $t$ & $g l$ & $p>0.05$ & $F$ & $p<0.05$ \\
\hline \multirow{2}{*}{$\begin{array}{c}\text { Psicoterapia Breve } \\
\text { Espejo }\end{array}$} & $\begin{array}{l}\text { Asociación en } \\
\text { positivo }\end{array}$ & Postest & 3.42 & 24 & 0.069 & 9.37 & 0.022 \\
\hline & Esfuerzo & Postest & 3.32 & 26 & 0.068 & 22.32 & 0.019 \\
\hline \multirow{2}{*}{$\begin{array}{l}\text { Estrategia de } \\
\text { aprendizaje } \\
\text { de Mapas } \\
\text { Conceptuales }\end{array}$} & $\begin{array}{c}\text { Elaboración de } \\
\text { anclaje }\end{array}$ & $\begin{array}{c}\text { Seguimiento seis } \\
\text { meses }\end{array}$ & 2.05 & 32 & 0.098 & $18 . .25$ & 0.031 \\
\hline & Planificación & $\begin{array}{c}\text { Seguimiento } 12 \\
\text { meses }\end{array}$ & 1.98 & 10 & 0.07 & 9.34 & 0.035 \\
\hline
\end{tabular}


5. Se realizó la comparación de número de estudiantes irregulares y promedio $<7.6$ con los estudiantes regulares que pasaron a ser y promedio semestral $>7.6$ de las 10 carreras (ver Tabla 5).
6. Se realizó un concentrado de número de estudiantes intervenidos con ambos procedimientos en las cuatro evaluaciones para observar el aumento de promedios de las 10 carreras (ver Tabla 6).

Tabla 5. Cantidad de estudiantes con promedios $<.76$ e irregulares en cuatro evaluaciones

\begin{tabular}{|c|c|c|c|c|c|c|c|c|c|c|c|}
\hline \multirow{3}{*}{$\begin{array}{c}\text { Evaluaciones } \\
\text { Estudiantes } \\
\text { intervenidos } \\
\text { Procedimientos }\end{array}$} & \multirow{2}{*}{\multicolumn{2}{|c|}{$\begin{array}{c}\text { Pre test } \\
\\
\text { Irregulares y } \\
\text { promedio }<7.6\end{array}$}} & \multicolumn{3}{|c|}{ Post test } & \multicolumn{3}{|c|}{ Seguimiento 6 meses } & \multicolumn{3}{|c|}{ Seguimiento 12 meses } \\
\hline & & & \multicolumn{2}{|c|}{$\begin{array}{c}\text { Irregulares y } \\
\text { promedio en } \\
\text { primer parcial }<7.6\end{array}$} & \multirow{2}{*}{$\begin{array}{c}\text { Irregulares } \\
\text { y promedio } \\
\text { en primer } \\
\text { parcial }>7.6\end{array}$} & \multicolumn{2}{|c|}{$\begin{array}{c}\text { Irregulares } \\
\text { y promedio } \\
\text { semestral }<7.6\end{array}$} & \multirow{2}{*}{$\begin{array}{c}\text { Regulares } \\
y \\
\text { promedio } \\
\text { semestral } \\
>7.6\end{array}$} & \multicolumn{2}{|c|}{$\begin{array}{l}\text { Irregulares y } \\
\text { promedio anual } \\
<7.6\end{array}$} & \multirow{2}{*}{$\begin{array}{c}\text { Regulares } \\
y \\
\text { promedio } \\
\text { anual }>7.6\end{array}$} \\
\hline & 2(EAMC) & 1 (PBE) & $2(\mathrm{EAMC})$ & 1 (PBE) & & 2(EAMC) & $1(\mathrm{PBE})$ & & 2(EAMC) & 1 (PBE) & \\
\hline LAF & 10 & 10 & 9 & 8 & 3 & 6 & 6 & 8 & 6 & 4 & 10 \\
\hline LEC & 14 & 13 & 12 & 10 & 5 & 11 & 10 & 6 & 10 & 7 & 10 \\
\hline LRI & 18 & 17 & 16 & 16 & 3 & 15 & 15 & 5 & 12 & 8 & 15 \\
\hline LCN & 30 & 30 & 28 & 22 & 10 & 22 & 21 & 17 & 20 & 18 & 22 \\
\hline LIA & 37 & 38 & 36 & 36 & 3 & 36 & 36 & 3 & 35 & 30 & 10 \\
\hline LDE & 40 & 41 & 38 & 38 & 5 & 40 & 39 & 2 & 38 & 33 & 10 \\
\hline IIN & 11 & 11 & 8 & 4 & 10 & 7 & 4 & 11 & 6 & 1 & 15 \\
\hline ISC & 39 & 40 & 35 & 35 & 9 & 34 & 30 & 15 & 30 & 22 & 27 \\
\hline ICO & 17 & 18 & 15 & 15 & 5 & 15 & 13 & 7 & 9 & 9 & 17 \\
\hline LAM & 25 & 24 & 18 & 14 & 17 & 16 & 16 & 17 & 12 & 11 & 26 \\
\hline TOTAL & 241 & 242 & 215 & 198 & 70 & 202 & 190 & 91 & 178 & 143 & 162 \\
\hline $\begin{array}{l}\text { Porcentaje a la } \\
\text { población de } \\
2510\end{array}$ & $9.60 \%$ & $9.64 \%$ & $8.57 \%$ & $7.89 \%$ & $2.79 \%$ & $8.05 \%$ & $7.57 \%$ & $3.63 \%$ & $7.09 \%$ & $5.7 \%$ & $6.4 \%$ \\
\hline $\begin{array}{c}\text { Porcentaje a } \\
\text { la muestra de } \\
483\end{array}$ & $49.90 \%$ & $50.1 \%$ & $44.50 \%$ & $41.0 \%$ & $14.50 \%$ & $41.80 \%$ & $39.3 \%$ & $18.80 \%$ & $36.90 \%$ & $29.6 \%$ & $33 . \%$ \\
\hline $\begin{array}{l}\text { Impacto de los } \\
\text { procedimientos }\end{array}$ & & & $5.4 \%$ & $9.1 \%$ & & $8.1 \%$ & $10.8 \%$ & & $13 \%$ & $20.5 \%$ & \\
\hline $\begin{array}{l}\text { 2(EAMC), Estr } \\
\text { Financiera; LEC } \\
\text { Licenciado en I } \\
\text { Computaciona }\end{array}$ & egia de $A$ & ndizaje & e Mapas & oncep & ales; 1(PB & sicotera & Breve & рејо. LA & _icenciac & $\begin{array}{l}\text { en Ac } \\
\text { tadu } \\
\text { en Si }\end{array}$ & $\begin{array}{l}\text { ría } \\
\text { LIA, } \\
\text { nas }\end{array}$ \\
\hline
\end{tabular}


Tabla 6. Impacto de ambos procedimientos observados en los promedio por carrera en cuatro evaluaciones

\begin{tabular}{|c|c|c|c|c|c|}
\hline Carreras & $\begin{array}{c}\text { Promedios } \\
\text { semestral } \\
\text { Evaluación Pretest }\end{array}$ & $\begin{array}{c}\text { Promedio parcial } \\
\text { Evaluación Post } \\
\text { test }\end{array}$ & $\begin{array}{l}\text { Promedio } \\
\text { semestral } \\
\text { Evaluación } \\
\text { Seguimiento a } \\
\text { seis meses }\end{array}$ & $\begin{array}{l}\text { Promedio anual } \\
\text { Evaluación } \\
\text { Seguimiento } 12 \\
\text { meses }\end{array}$ & $\begin{array}{l}\text { Impacto de los } \\
\text { procedimientos }\end{array}$ \\
\hline $\mathrm{LAF}$ & 6.71 & 7.553 & 7.321 & 7.55 & 1.125 \\
\hline LEC & 6.84 & 7.575 & 6.893 & 7.45 & 1.090 \\
\hline LRI & 7.18 & 7.805 & 7.355 & 7.64 & 1.064 \\
\hline LCN & 6.74 & 7.079 & 7.322 & 7.63 & 1.131 \\
\hline LIA & 6.87 & 7.536 & 7.151 & 7.75 & 1.129 \\
\hline LDE & 6.83 & 7.471 & 7.529 & 7.54 & 1.104 \\
\hline IIN & 6.82 & 7.543 & 7.356 & 7.39 & 1.083 \\
\hline ISC & 6.81 & 7.3 & 7.348 & 7.40 & 1.086 \\
\hline ICO & 6.75 & 7.694 & 7.357 & 7.31 & 1.084 \\
\hline LAM & 6.81 & 7.483 & 7.283 & 7.52 & 1.105 \\
\hline $\begin{array}{l}\text { Promedio global } \\
\text { de la nuestra }\end{array}$ & 68.35 & 75.04 & 72.92 & 75.19 & 1.100 \\
\hline $\begin{array}{l}\text { LAF, Licenciado en } \\
\text { LCN, Licenciado e } \\
\text { Ingeniería Industri } \\
\text { en Administración }\end{array}$ & $\begin{array}{l}\text { ctuaría Financiera; } \\
\text { Contaduría; LIA, Li } \\
\text { ISC, Ingeniería en }\end{array}$ & $\begin{array}{l}\text { C, Licenciado en } \\
\text { ciado en Inform } \\
\text { temas Computac }\end{array}$ & $\begin{array}{l}\text { nomía; LRI, Li } \\
\text { Administrativ } \\
\text { ales; ICO, Inge }\end{array}$ & $\begin{array}{l}\text { ado en Relacione } \\
\text { E, Licenciado en } \\
\text { a en Computacic }\end{array}$ & $\begin{array}{l}\text { ternacionales; } \\
\text { recho, IIN, } \\
\text { AM, Licenciado }\end{array}$ \\
\hline
\end{tabular}

\section{DISCUSIÓN}

Como ya se ha referido en líneas anteriores, algunos de los grandes problemas que enfrentan las instituciones de educación superior oficiales son el bajo rendimiento, irregularidad, deserción, el rezago estudiantil, que reflejan el nivel de la eficiencia terminal, lo que de alguna manera también afecta al C.U UAEM Valle de México.

Por lo que en este estudio se implementaron dos procedimientos: Psicoterapia Breve Espejo 1(PBE) y Estrategia de Aprendizaje de Mapas Conceptuales 2 (EAMC), con los que se impactó a 162 estudiantes con bajos promedios e irregulares. De la población de 483 se impacto el $20.5 \%$ en 1 (PBE) y el $13 \%$ en 2 (EAMC). Para tener una visión más detallada del impacto de los procedimientos nos dimos a la tarea de analizar las escalas que los medían. Observamos que:

En el procedimiento $1(\mathrm{PBE})$ se presenta una disminución significativa de estudiantes en los trastornos emocionales de Ansiedad y Baja autoestima. También se observa un aumento significativo en estrategias motivacionales de aprendizaje, como en Asociación en Positivo y Auto-esfuerzo, lo que ayuda a regularizar y a aumentar el promedio a 97 estudiantes, ocupando el $62 \%$ del impacto de intervenciones.

Es oportuno comentar la existencia de diversos estudios sobre la estabilidad emocional y su repercusión en el rendimiento académico, como los realizados por González-Forteza, et al., (2008); Campo-Cabal y Gutiérrez (2001).

En el procedimiento 2 (EAMC) disminuyó significativamente el número de estudiantes con trastornos de ansiedad como elaboración de anclaje y planificación. Esto es posible porque este procedimiento obtiene aprendizaje significativo, a través de comprender cómo relacionar los nuevos conocimientos, de manera organizada y sustancial, encadenándolos con aprendizajes 
previos. Esto le da confianza a los estudiantes para realizar sus actividades académicas, como lo mencionan Ausubel, Novak y Hanesian en 1997, lo que ayuda a conservar el aumento de su rendimiento escolar. En este procedimiento se reporta el impacto en 60 estudiantes regulares y regular rendimiento, lo que ocupa el $38 \%$.

Teniendo presente la anterior aseveración, es lógico pensar que las estrategias de aprendizaje deben de tener más impacto en los promedios bajos y en la irregularidad estudiantil que un tratamiento psicoterapéutico, pero en este estudio se observó que existe un avance semejante en evaluaciones pro test y seguimiento de seis meses en ambos procedimientos, pero que en el tratamiento psicoterapéutico existe una diferencia significativa en la evaluación seguimiento de 12 meses; lo que quiere decir que el Tratamiento Psicoterapia

\section{REFERENCIAS}

Adlaf, M., Gliksman, L.; Demers, A. \& Newton-Taylor, B. (2001). The prevalence of elevated psychological distress among canadian undergraduates: findings from the 1998 Canadian Campus Survey. J Am Coll Health, 50 (2), 67-72. Recuperado el 27 de Mayo de 2010 de http://www.biomedsearch.com/nih/prevalence-elevated-psychological-distress-among/11590985.html

Amézquita, M., González, P. \& Zuluaga, M. (2000). Prevalencia de la depresión, ansiedad y comportamiento suicida en la población estudiantil de pregrado de la Universidad de Caldas. Revista Colombiana de Psiquiatría, 4,341-356. Recuperado el 25 septiembre de 2010 de http://redalyc.uaemex.mx/ pdf/806/80632404.pdf.

Arco, J. L, López, S., Heilborn, V. \& Fernández, F. (2005). Terapia breve en estudiantes universitarios con problemas de rendimiento académico y ansiedad: eficacia del modelo "La Cartuja". International Journal of Clinical and Health Psychology, 5, 589-608. Recuperado el 27 de Mayo de 2010 de: redalyc.uaemex.mx/redalyc/pdf/337/33705310.pdf

Ausubel, D. P.; Novak, J. D. \& Hanesian, H. (1997). Psicología educativa. Un punto de vista cognoscitivo. (10ª ed.). México: Trillas. /
Breve Espejo tiene mayor impacto a largo plazo. Esto puede deberse a que los estudiantes en el bloque de integración realizan diálogos con su propia imagen, confrontándose consigo mismos, dándose cuenta de la sintonía que existe entre mente y cuerpo (Campos \& Gutiérrez, 2001; González \& Turmbull, 2003, 2007; González \& López, 2009:). En el bloque 2 se interviene en los procesos mentales asociados a la Adquisición, Codificación y Recuperación, lo que le va contribuir a mejorar su rendimiento escolar (Borkowski, 1995; Campos y Gutiérrez, 2001). Ahora es importante hacer un análisis por carrera y actividades académicas, como lo ejecutan Amézquita, González y Zuluaga (2000) con ambas estrategias, y en el cual se observó: la estrategia 1(PNE) obtiene un efecto en 10 estudiantes de 11 , o sea que $91 \%$ en la Carrera de Ingeniería Industrial son recuperados.

Borkowski, J. G. (1995). Sings of intelligence. Orlando, Fl.: Academic Press.

Campos, G. \& Gutiérrez, S.J. (2001). Psicopatología en estudiantes universitarios. Revista Colombiana de Psiquiatría, 30 (4). Recuperado el 27 de Mayo de 2010 de: redalyc.uaemex.mx/redalyc/pdf/806/80630402.pdf Cañas, A.J., Ford, K.M., Hayes, P.J., Reichherzer, T., Suri, N., Coffey, J., et al. (2002). Aprendizaje a través de mapas conceptuales. Institute for Human and Machina Cognition. University of West. Recuperado el 27 de Mayo de 2010 de: http://www.ilhn.com/datos/archives/000052.php

Cuevas, A. (2003). Propuesta de aplicación de los mapas conceptuales en un modelo pedagógico semipresencial. Instituto Superior Politécnico "José Antonio Echeverría”. Revista Iberoamericana de Educación. Recuperado el 27 de Mayo de 2010 de: http://www. rieoei.org/deloslectores/493Cuevas.PDF

Declaración de Helsinki de la Asociación Médica Mundial (2008). ARBOR Ciencia, Pensamiento $y$ Cultura CLXXXIV 730 marzo-abril 349-352: autor Recuperado el 8 de Junio de 2010 de: http://www. censida.salud.gob.mx/descargas/etica/declaracion_ Helsinki.pdf 
Delors, J. (1997). La educación encierra un tesoro. Informe a la UNESCO de la Comisión Internacional sobre la educación para el siglo XXI. Francia: Santillana, Ediciones UNESCO.

Estrada, V. \& Febles, J.P. (2002). La gestión del conocimiento y las universidades. VIII Convención de Informática en la Educación. [Documentos de Congreso]. La Habana, Cuba. Recuperado el 27 de Mayo de 2010 de: http://espejos.unesco.org.uy/simplac2002/Ponencias/ Inforedu/IE118\%20Vivian\%20Estrada\%20Senti.doc

Febles, J. P. \& Estrada, V. (2002). Aplicaciones de la inteligencia artificial. Parte III. Mapas conceptuales. (1 ${ }^{\mathrm{a}}$ ed.) México: Universidad de Guadalajara, p. 400.

González Forteza C., Arana Q.D., \& Jiménez J.A, (2008). Problemática suicida en adolescentes y su contexto escolar: Vinculación autogestiva con los servicios de salud. Salud Mental 31(1), 23-27. Recuperado el 27 de Septiembre de 2010 de: http://redalyc.uaemex. $\mathrm{mx} / \mathrm{pdf} / 582 / 58231104$.pdf.

González, C., García, G., Medina-Mora, M., Sánchez, M. (1998). Indicadores psicosociales predictores de ideación suicida en dos generaciones de estudiantes universitarios. Salud Mental, 21(3), 1-9.

González, J. \& López, S. (2008). Tratamientos para el trastorno por consumo de alcohol en trabajadores del sector salud. Revista de Psicología Social Aplicada, 18(2), 127-149. Recuperado el 26 abril de 2011: http://dialnet.unirioja.es/servlet/listaarticulos?tipo _busqueda=EJEMPLARyrevista_busqueda= 1209yclave_busqueda $=245002$

González, J. \& Turmbull, P. (2003). Selection of psychotherapeutic treatment for adjustment disorder with depressive mood due to acute myocardial infarction. Archives of Medical Research, 2003: 34 (4), 298-304. Recuperado el 26 abril de 2011: http://www.arcmedres.com/article/S0188-4409(03)00051-1/abstract

González, J. \& Turmbull, P. (2007). Trastornos mentales en pacientes post-infarto. Revista de psicología y ciencias sociales UNAM, 9 (1), 32-24. Recuperado el 23 de octubre de 2010 de: http://www.catalogoderevistas.unam.mx/interiores/p/psic_cs.html

Hernández, P. \& García, L.A. (1994). A framework for teaching learning strategies and study skills in the curriculum. En H. Ross (Ed) Designing for learning pp. 30-4, Londres: Kogan Page.
Hernández, P. \& García, L.A. (1997). Enseñar a pensar. Un reto para los profesores. Santa Cruz de Tenerife: Tafor ediciones.

Lucio G-M. E. (1995). Inventario multifacético de la personalidad de Minnesota-2. México: Manual Moderno.

Martín, E., García, L.A., Torbay, A. \& Rodríguez, T. (2007) Estructura factorial y fiabilidad de un cuestionario de estrategias de aprendizaje en universitarios: CEA-U. Anales de Psicología, 23(1),6. Recuperado el 27 de Mayo de 2010 de: redalyc.uaemex.mx/redalyc/ pdf/167/16723101.pdf

Novak, J. D. (1991). Ayudar a los alumnos a aprender como aprender. La opinión de un profesor-investigador en Enseñanza de las Ciencias, 9, 3, p.p. 215-227. Recuperado el 27 de Mayo de 2010 de: dialnet.unirioja.es/servlet/articulo? codigo $=94518$

Piña J.M., Furlan A. \& Suñido L. (2003). La investigación educativa en México: Acciones actores y prácticas educativas. Consejo Nacional de la Investigación Educativa en México: México, pp. 326-327.

Programas Institucionales de Tutoría. (2002). Una propuesta de la ANUIES para su organización y funcionamiento en las Instituciones de Educación Superior. Seminario sobre estándares de calidad para instituciones de educación superior. D.F., México: Asociación Nacional de Universidades en Instituciones de Educación Superior. Recuperado el 29 de Mayo de 2010 de: http://dape.pucp.edu.pe/node/630

Romo, A. (2005). La incorporación de los programas de tutoría en las instituciones de educación superior D.F., México: Asociación Nacional de Universidades e Instituciones de Educación Superior, p.p. 23. Recuperado el 27 de Mayo de 2011 de: http://www. anuies.mx/e_proyectos/pdf/parte1.pdf

Universidad Autónoma del Estado de México, Reglamento de Facultades y Escuelas Profesionales (2009), Estado de México: Autor. Recuperado el 17 de octubre 2010 de: http://scfi.uaemex.mx/ ragarcia/docs-uap/ ReglamentoFacultadesEscuelasProfesionales.pdf

Valle, A., Cabanach, R.G., Rodríguez, S., Núñez, J.C. y González, J.A. (2006). Metas académicas, estrategias cognitivas y estrategias de autorregulación del estudio. Psicothem, 18, 165-170. Recuperado el 27 de Mayo de 2010 de: http://www.psicothema.com/ pdf/3193.pdf 
Wolters, C.A. (2003). Regulation of motivation: evaluating and underemphasized aspect of self-regulated learning. Educational Psychologist, 38, 189-205. Recuperado el 29 Mayo de 2011 de: http://www.informaworld.com/smpp/content $\sim \mathrm{db}=\mathrm{all} \sim$ content $=\mathrm{a} 7$ 84751361 frm=titlelink

Fecha de recepción: Diciembre 2010 Fecha de publicación: Marzo 2011 